\title{
PRIMER REGISTRO DE RHIPICEPHALUS SANGUINEUS (LATREILLE, 1806) (ACARI: IXODIDAE) EN RATTUS NORVEGICUS (MAMMALIA: RODENTIA) EN CHILE
}

\section{FIRST RECORD OF RHIPICEPHALUS SANGUINEUS (LATREILLE, 1806) (ACARI: IXODIDAE) IN RATTUS NORVEGICUS (MAMMALIA: RODENTIA) IN CHILE}

\author{
Daniel González-Acuña ${ }^{1}$, José M. Venzal ${ }^{2}$ \& Alberto A. Guglielmone ${ }^{3}$ \\ 'Facultad de Medicina Veterinaria, Universidad de Concepción, Casilla 537, Chillán, Chile. \\ ${ }^{2}$ Departamento de Parasitología Veterinaria, Facultad de Veterinaria, Universidad de la República, Av. Alberto \\ Lasplaces 1550, Montevideo, Uruguay. \\ ${ }^{3}$ Instituto Nacional de Tecnología Agropecuaria, CC 22, CP 2300 Rafaela, Santa Fe, Argentina.
}

\begin{abstract}
RESUMEN
Se registra por primera vez en Chile a Rhipicephalus sanguineus parasitando a un huarén (Rattus norvegicus) en la ciudad de Chillán. Se discute la importancia sanitaria hipotética de este hallazgo.

Palabras Claves: Rhipicephalus sanguineus, Rattus norvegicus, Ixodidae, Chile.
\end{abstract}

\section{ABSTRACT}

A male Rhipicephalus sanguineus was found on a Rattus norvegicus in the city of Chillán (Chile). This is the first record on this host in Chile. The hypothetical sanitary importance of the finding is discussed.

KeYwords: Rhipicephalus sanguineus, Rattus norvergicus, Ixodidae, Chile.

\section{INTRODUCCION}

Rhipicephalus sanguineus (Latreille 1806) es una garrapata que se ha observado en diferentes mamíferos (Alcaíno 1985), pero su hospedador preferencial es el perro. Esta garrapata es de origen africano, desde donde alcanzó la más amplia distribución en el mundo (Keirans 1992). Su hallazgo en el hombre fue registrado en varios países, incluido Chile (Schenone 1996), donde se determinó que el 2,2\% de los casos de picaduras de artrópodos en humanos en el país era debido a
$R$. sanguineus, que, a su vez, causaba ocasionalmente una dermatitis pruriginosa severa en los niños. La picadura en los humanos se relaciona con la transmisión de rickettsias de importancia sanitaria (Burgdorfer 1975; Goddard 1989; Unver et al. 2001). Los hallazgos de $R$. sanguineus en Chile abarcan desde Viña del Mar (32 ${ }^{\circ} 59^{\prime} S$ $71^{\circ} 33^{\prime}$ W) (Alcaíno 1985) hasta Concepción (36 $51^{\circ} \mathrm{S} 72^{\circ} 59^{\prime} \mathrm{W}$ ) (Muñoz y Casanueva 2001) y, con la excepción ya mencionada de Schenone (1996), los hallazgos han sido realizados hasta la fecha sólo en perros. 


\section{MATERIAL Y METODOS}

El día 9 de enero de 2002 se capturó un huarén (Rattus norvegicus) macho adulto en el Campus Chillán de la Universidad de Concepción (36 $36 \mathrm{~S}$ $72^{\circ} 06^{\top} \mathrm{W}$ ), provincia de Nuble (VII Región), Chile, parasitado con una garrapata en la zona cervical. El ectoparásito fue conservado en alcohol al 70\%, para luego ser identificado siguiendo las pautas de Boero (1957). El ejemplar fue depositado en la colección del Departamento de Ciencias Pecuarias, Laboratorio de Zoología, Chillán, Universidad de Concepción.

\section{RESULTADOS}

La garrapata fue identificada como un macho de $R$. sanguineus. Este es el primer hallazgo de esta especie de garrapata en $R$. norvegicus para Chile.

\section{DISCUSION}

Existen escasos antecedentes del parasitismo por $R$. sanguineus en especies del género Rattus para el neotrópico. Fox (1950) observó a esta especie de garrapata en $R$. norvegicus y $R$. rattus en Puerto Rico; Thompson (1950) en $R$. rattus en Jamaica y Yoshizawa et al. (1996) observaron su presencia en $R$. norvegicus en Brasil. Aunque Fox (1950) considera que la tasa de infestación de Rattus con $R$. sanguineus es de bajo nivel y no tendría un rol de importancia en la transmisión del tifo murino en Puerto Rico, no puede ser descartado el flujo de microorganismos patógenos desde los roedores a los perros y viceversa, ciclo potencial donde el hombre podría ocasionalmente estar involucrado. El presente reporte es el primero en Chile en el que $R$. sanguineus parasita a un roedor, debido a lo cual sería de importancia considerarlos en las medidas de manejo y control de esta especie de garrapatas en perros. En cualquier caso es de interés un relevamiento de garrapatas en $R$. norvegicus para estimar la abundancia de $R$. sanguineus en este tipo de hospedador.

\section{BIBLIOGRAFIA}

Alcaíno, H. 1985. Antecedentes sobre la garrapata café del perro (Rhipicephalus sanguineus). Monografia Med. Vet. 7 (2), 48-55.

Boero, J.J. 1957. Las garrapatas de la República Argentina (Acarina: Ixodoidea). Depto. Edit. Univ. Buenos Aires, Buenos Aires, 113 pp.

Fox, I. 1950. The brown dog tick on rats in Puerto Rico. J. Econ. Entomol., 43: 722-723.

GodDARD, J. 1989. Focus of human parasitism by the brown dog tick, Rhipicephalus sanguineus (Acari: Ixodidae). J. Med. Entomol., 26: 628-629.

Muñoz, L.E. \& M.E. Casanueva. 2001. Ampliación de ámbito de la garrapata Rhipicephalus sanguineus (Acari: Ixodidae) en Chile. Rev. Biol. Trop., 49: 1285.

KeIrans, J.E. 1992. Systematics of the Ixodida (Argasidae, Ixodidae, Nuttalliellidae): an overview and some problems. In B. Fivaz, T. Petney and I. Horak (eds.). Tick vector biology. Medical and veterinary aspects. Springer-Verlag, Berlin, pp $1-21$.

Schenone, H. 1996. Diagnósticos hechos a 1.384 pacientes que consultaron por probable mordedura de arañas o picaduras de insectos. Bol. Chil. Parasitol., 51: 20-27.

Thompson, G.B. 1950. Ticks in Jamaica, B.W.I. Records and notes (including a summary of the distribution of the West Indian species). Ann. Mag. Nat. Hist. Ser. 12, 3: 220-229.

Unver, A., M. Perez, N. Orellana, H. Huang \& Y. Rikisha. 2001. Molecular and antigenic comparison of Ehrlichia canis isolates from dogs, ticks, and a human in Venezuela. J. Clin. Microbiol., 39: 2788-2793.

Yoshizawa, M.A.C., J.L. Souza, A. Bredt, D. Baggio. 1996. Ectoparásitos de Rattus norvergicus no Distrito Federal, Brasil. Rev. Bras. Parasitol. Vet., 5: 39-42. 